\title{
Estudios en el género Cortaderia (Poaceae). III. Cortaderia PERUVIANA, NUEVO SINÓNIMO DE C. HIERONYMI
}

\author{
DANIEL TESTONI ${ }^{1}$
}

\begin{abstract}
Summary: Studies in the genus Cortaderia (Poaceae) III. Cortaderia peruviana, a new synonym of C. hieronymi. Cortaderia hieronymi and C. peruviana have been considered as two different species. However, different authors differ in their morphological limits and their geographical distribution. A Principal Components Analysis based on characters traditionally used to delimit these species did not show discrete groupings for each taxon. In addition, the study of type species and their original descriptions, complemented with field studies, allows to establish that C. peruviana is part of the morphological variability of $C$. hieronymi; therefore, their synonymy is proposed herein. A detailed characterization and geographic distribution of the species is given.
\end{abstract}

Key words: Cortaderia, multivariate analysis, Lamprothyrsus, Poaceae, systematics.

Resumen: Cortaderia hieronymi y C. peruviana han sido consideradas como dos especies diferentes. Sin embargo, distintos autores difieren en sus límites morfológicos y en su distribución geográfica. Un análisis de componentes principales sobre la base de los caracteres tradicionalmente utilizados para delimitar estas especies no permitió formar grupos discretos para cada taxón. Además, el estudio de los ejemplares tipo y de las descripciones originales, complementado con estudios de campo, permite establecer que C. peruviana es parte de la variabilidad morfológica de C. hieronymi; por lo tanto, se propone su sinonimia. Se amplía la caracterización y distribución geográfica de la especie.

Palabras clave: Análisis multivariado, Cortaderia, Lamprothyrsus, Poaceae, sistemática.

\section{INTRODUCCIÓN}

Recientemente se han dado a conocer distintas sinonimias para el género Cortaderia Stapf (Testoni \& Villamil, 2014; Testoni, 2015). Continuando con estos estudios, se han hallado novedades en la sistemática de las especies que componían su género hermano, Lamprothyrsus Pilg.

La afinidad entre Cortaderia y Lamprothyrsus ha sido ampliamente discutida. Pilger (1906) los separa en base a unos pocos caracteres exomorfológicos de las glumas y lemas, y Conert (1961) aporta diferencias en la anatomía foliar. Sin embargo, Connor \& Dawson (1993)

\footnotetext{
1 Herbario BBB, Departamento de Biología, Bioquímica y Farmacia, Universidad Nacional del Sur. San Juan 670, Bahía Blanca (8000), Argentina. Becario CONICET; daniel.testoni@uns.edu.ar
}

indican que coiciden en su sistema reproductivo: "Asexuality in females evolved in Lamprothyrsus and Cortaderia in the identical form of autonomous (nonpseudogamous) apospory after dioecism, subdioecism, or gynodioecism had become fixed as gender dimorphisms. Further, megagametogenesis is similar in both; the embryo sac, nucellar in origin, is commonly 6-nucleate, since one synergid and one antipodal are absent". Barker et al. (2003) en base a estudios filogenéticos considera que ambos géneros forman un grupo monofilético. Finalmente, Linder et al. (2010) proponen su sinonimia.

Pilger (1906) funda el género monotípico Lamprothyrsus, basado en Triraphis hieronymi Kuntze, con cinco variedades, citando los materiales provenientes de la Argentina (Córdoba y Jujuy) y Bolivia. Posteriormente, Hitchcock (1923) describe L. peruvianus en base a materiales de Perú, y Conert (1961) describe L. venturii, sobre un material de la Argentina (Tucumán). Bernardello 
(1979), en su revisión del género Lamprothyrsus para la Argentina, propone la sinonimia de las cinco variedades de L. hieronymi y de $L$. venturii.

La distribución geográfica de estos taxones difiere de acuerdo al criterio de los diferentes autores. Hitchcock (1927) y Bernardello (1979) estiman que Lamprothyrsus hieronymi crece desde Bolivia y hasta el centro de la Argentina, en tanto que L. peruvianus, lo hace desde Ecuador hasta Perú. Tovar (1993), cita a L. hieronymi para la Argentina, Bolivia y Perú y a L. peruvianus para Bolivia, Perú y Ecuador. Para la flora de Ecuador, Lægaard (1997) considera sólo la presencia de $L$. peruvianus; sin embargo, menciona la existencia de materiales con características que coinciden con la especie hermana, y pone en duda la identidad de estos taxones.

Como parte de la revisión integral del género Cortaderia, este trabajo tiene por objetivo discutir la validez de los binomios de $C$. hieronymi y $C$. peruviana.

\section{Material y Método}

\section{Material de estudio}

Se analizó un gran número de ejemplares herbario de Cortaderia hieronymi y de C. peruviana coleccionados en la Argentina, Bolivia, Perú y Ecuador, depositados en los herbarios BA, BAA, BBB, CORD, LOJA, QCA y SI (Thiers, 2015). Para el estudio comparativo de la variación morfológica de estas especies, se seleccionaron 27 ejemplares: 18 de C. hieronymi y 9 de C. peruviana. Los materiales estudiados se detallan bajo material examinado, del capítulo "tratamiento taxonómico".

\section{Caracteres morfológicos y análisis estadístico}

Los caracteres analizados se muestran en la Tabla 1. Se seleccionaron 19 variables exomorfológicas relacionadas con estructuras reproductivas utilizadas tradicionalmente para la caracterización de $C$. hieronymi y C. peruviana (Pilger, 1906; Hitchcock, 1923; Bernardello, 1979; Tovar, 1993; Lægaard,

Tabla 1. Descripción y codificación de los caracteres utilizados en el análisis de componentes principales.

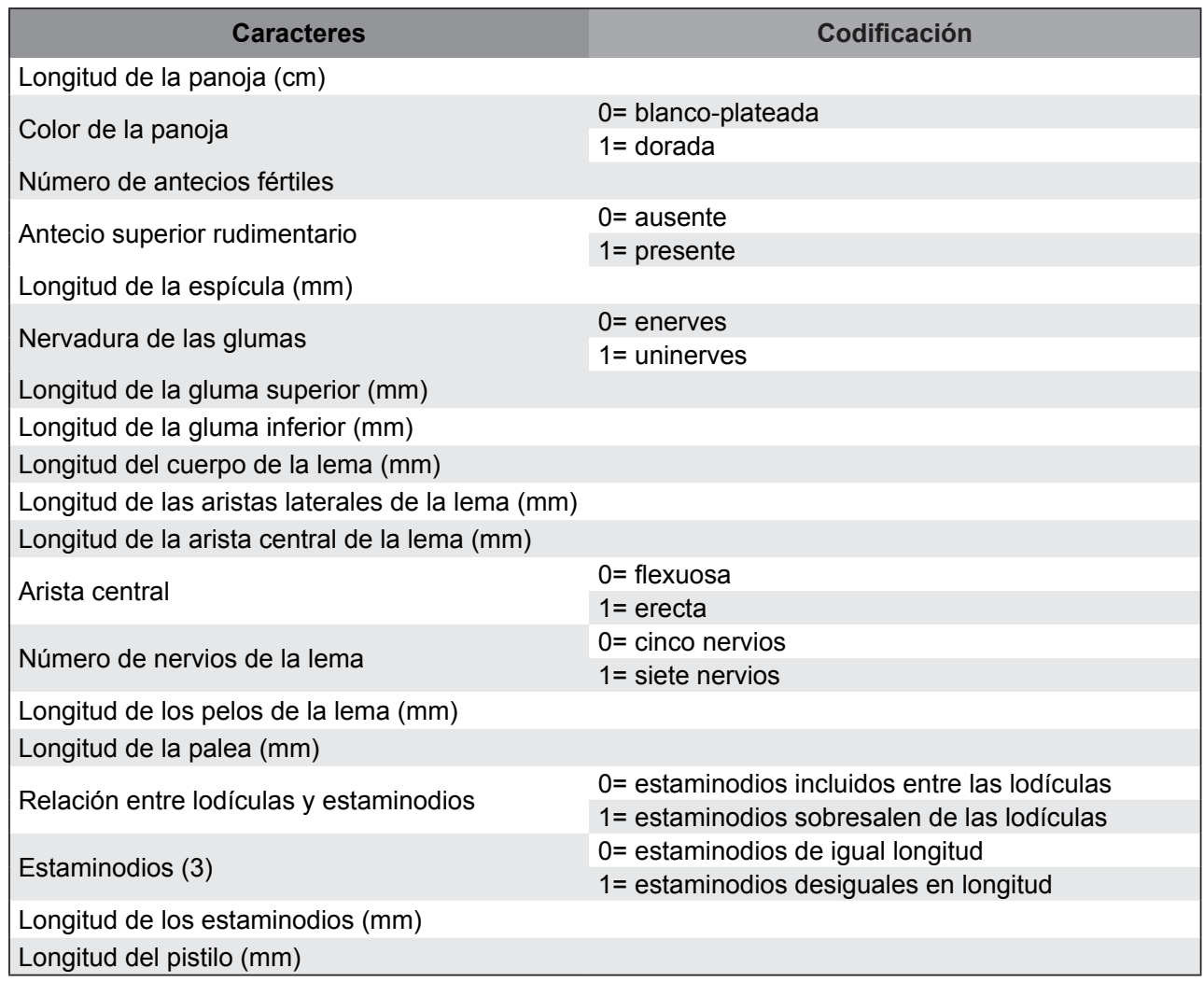




\section{Testoni - Cortaderia peruviana, nuevo sinónimo de C. hieronymi}

1997). Siete variables son cualitativas doble estado, 11 cuantitativas de variación continua y una cuantitativa de variación discreta. De cada ejemplar se estudiaron 10 espículas tomadas al azar de la panoja. Las medidas corresponden al antecio basal; cuando los estaminodios son desiguales, se midió el mayor. A los datos obtenidos de variación continua se les calculó el promedio en tanto que a los de variación discreta y cualitativos se les calculó la moda. Los datos fueron incluidos en un análisis de componentes principales (ACP) a partir de una matriz de similitud basada en el coeficiente de correlación de Pearson, previamente estandarizados: a cada valor se le sustrajo la media y dividió por el desvío estándar. El ACP se realizó utilizado el programa estadístico InfoStat (Di Rienzo et al., 2008) y el gráfico mediante R (R Core Team, 2013).

Complementariamente, se evaluó la significancia de las diferencias en los valores medios obtenidos entre $C$. hieronymi y C. peruviana para los caracteres cuantitativos de variación continua, mediante la prueba no paramétrica de Mann-Whitney, utilizando el programa estadístico SPSS 15.0.1 (SPSS Inc., 2006).

\section{Nomenclatura}

Se revisó críticamente la nomenclatura de ambas entidades y sus sinónimos (Index Kewensis, 1997; IPNI, 2010), según las normas establecidas en el Código Internacional de Nomenclatura Botánica
(McNeill et al., 2012). Se estudiaron los ejemplares tipo de estos binomios a través de la foto del ejemplar, disponible en JSTOR (http://plants.jstor. org) o en las bases de datos de los herbarios. Se indica "cb" para los códigos de barra.

\section{Resultados}

En la Fig. 1 se muestra el resultado del ACP que incluye los ejemplares previamente determinados como Cortaderia hieronymi y C. peruviana. Los primeros tres componentes incluyen el $49 \%$ de la variabilidad total. El ACP no permite separar estas dos especies sobre la base de sus caracteres morfológico-reproductivos.

En la Tabla 2 se muestran los resultados del análisis no paramétrico de Mann-Whitney. No se hallaron diferencias significativas $(p>0,05)$ en los valores medios obtenidos entre $C$. hieronymi y $C$. peruviana para ninguna de las variables consideradas.

\section{Discusıón}

El sistema reproductivo de los taxones incluidos en este trabajo es predominantemente asexual, apomíctico, con formación de cariopsis sin la intervención de polen (Connor \& Dawson, 1993);

Tabla 2. Resultados de la prueba no paramétrica de Mann-Whitney para distintos caracteres morfológicos entre Cortaderia hieronymi y C. peruviana: valores medios y desvío estándar, estadístico de Mann-Whitney $(U)$ y significancia entre las medias $(p)$.

\begin{tabular}{|c|c|c|c|c|}
\hline Caracteres morfológicos & Cortaderia hieronymi & Cortaderia peruviana & $\mathbf{U}$ & p \\
\hline Longitud de la panoja (cm) & $31,66( \pm 11,24)$ & $40,77( \pm 11,25)$ & 44 & 0,053 \\
\hline Longitud de la espícula (mm) & $34,66( \pm 6,57)$ & $35,22( \pm 5,04)$ & 77 & 0,834 \\
\hline Longitud de la gluma superior (mm) & $8,88( \pm 0,75)$ & $8,66( \pm 0,86)$ & 67 & 0,441 \\
\hline Longitud de la gluma inferior (mm) & $7,77( \pm 0,80)$ & $7,66( \pm 1,11)$ & 69 & 0,500 \\
\hline Longitud del cuerpo de la lema (mm) & $3,69( \pm 0,52)$ & $3,66( \pm 0,50)$ & 75 & 0,741 \\
\hline Longitud de las aristas laterales de la lema (mm) & $19,05( \pm 4,35)$ & $19,33( \pm 2,17)$ & 77 & 0,834 \\
\hline Longitud de la arista central de la lema (mm) & $26,05( \pm 5,42)$ & $26,11( \pm 2,20)$ & 76,5 & 0,812 \\
\hline Longitud de los pelos de la lema (mm) & $5,05( \pm 0,63)$ & $5,01( \pm 0,70)$ & 77,5 & 0,838 \\
\hline Longitud de la palea (mm) & $3,64( \pm 0,28)$ & $3,61( \pm 0,41)$ & 80,5 & 0,977 \\
\hline Longitud de los estaminodios (mm) & $1,1( \pm 0,45)$ & $1,22( \pm 0,44)$ & 68 & 0,458 \\
\hline Longitud del pistilo (mm) & $3,14( \pm 0,58)$ & $3,38( \pm 0,54)$ & 60 & 0,254 \\
\hline
\end{tabular}




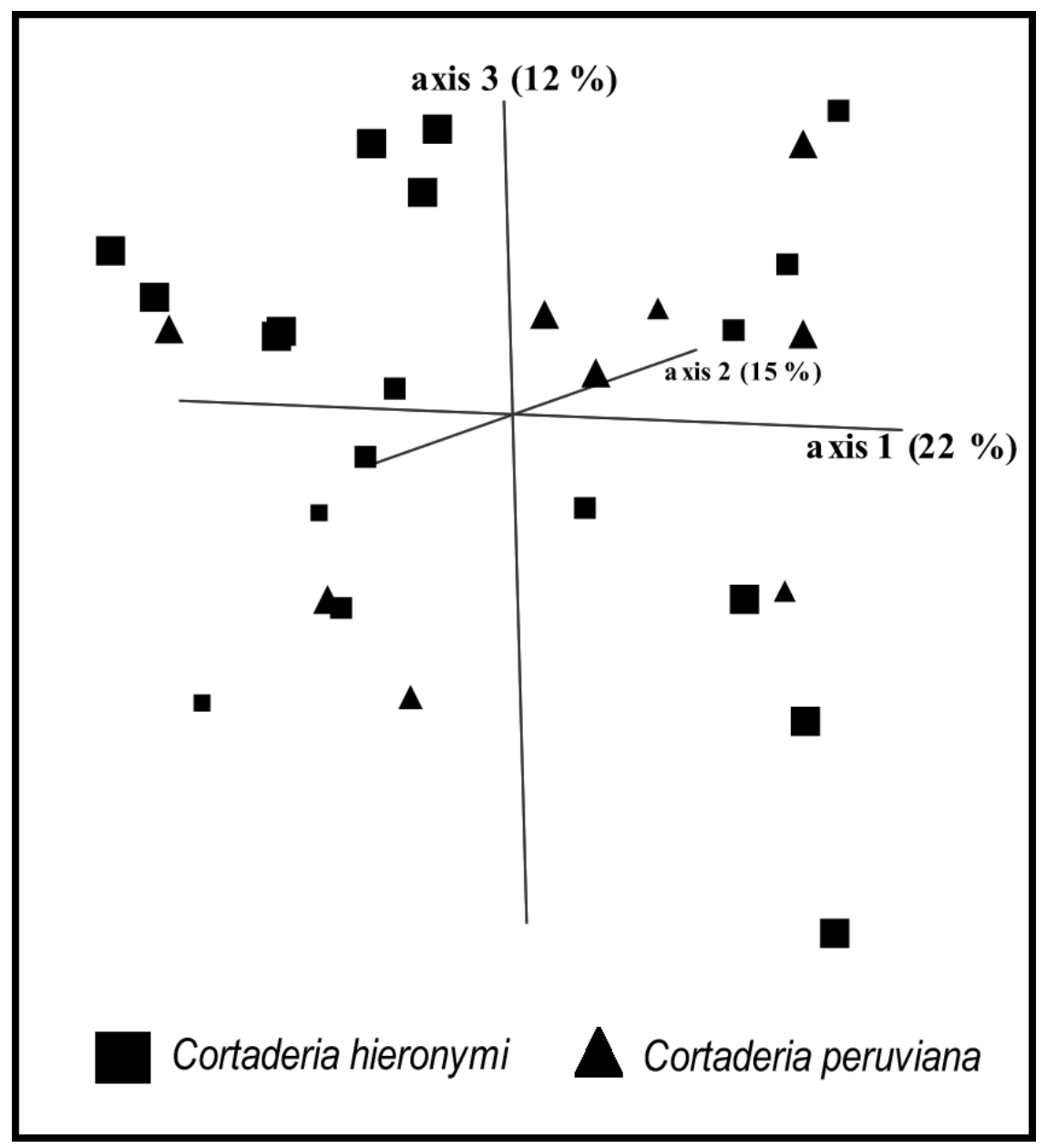

Fig. 1. Análisis de componentes principales. Representación en los primeros tres ejes del ordenamiento de ejemplares previamente determinados como de Cortaderia hironymi y de C. peruviana.

solo unos pocos ejemplares son estaminados, con anteras fértiles (Bernardello, 1979). Lægaard (1997) sostiene que "the whole genus with its three species and several varieties may constitute a group of apomictic microspecies". Esto se traduce en poblaciones con individuos muy homogéneos entre sí, como ocurre en otras especies del género (Testoni \& Villamil, 2014), pero la variabilidad entre poblaciones es suficientemente perceptible e incluso ha permitido la descripción de distintas variedades.

Del estudio crítico de las descripciones originales y otros trabajos clásicos que incluyen a Cortaderia hieronymi y a $C$. peruviana se encuentra que las diferencias entre ambos taxones son únicamente de naturaleza cuantitativa: Bernardello (1979), luego de analizar un gran número de ejemplares de las distintas variedades de Lamprothyrsus hieronyumi y de L. venturii (pero no de L. peruvianus), observó que la "altura de las plantas, coloración y dimensión de las panojas, nerviación de las glumas, número de flores por espiguilla, longitud de las aristas de la lemma, sólo para citar algunos de ellos, son caracteres que varían hasta en el mismo ejemplar". Hitchcock (1923), al describir Lamprothyrsus peruvianus, indicó que "This species differs from L. hieronymi (Kuntze) Pilger in the more dense purplish panicle and the longer glumes and lemmas...The awns are white and flexuous, while in L. peruvianus they are purple and nearly 


\section{Testoni - Cortaderia peruviana, nuevo sinónimo de C. hieronymi}

straight”. Posteriormente, Hitchcock (1927) agrega que $L$. hieronymi presenta "the florets smaller, the awns loosely twisted". En base a estas revisiones, Tovar (1993), en su tratamiento para la flora de Perú, agrega que ambos taxones se diferencian por el tamaño del cuerpo la lema ( $3 \mathrm{~mm}$ en L. hieronymi y $4 \mathrm{~mm}$ en $L$. peruvianus). Sin embargo, mediante el análisis no paramétrico se determinó que las diferencias en los caracteres que eran utilizados para diferenciar a estos taxones resultaron ser estadísticamente no significativas (Tabla 2).

Entre los ejemplares analizados del complejo Cortaderia hieronymi-peruviana, se hallaron muestras con características morfológicas similares entre sí, pero inconsistentes con en su distribución geográfica. A modo de ejemplo, un material de la Argentina (Burkart 13041) citado por Bernardello (1979) como L. hieronymi, es prácticamente imposible de diferenciar de otro (Lægaard 19124), determinado como L. peruvianus por Lægaard para el proyecto "Flora of Ecuador". Contrariamente, Lægaard (1997) cita el ejemplar Lægaard 70267, también de Ecuador, con características mencionadas en la literatura para L. hieronymi.

Con el auxilio del ánalisis multivariado se pudo determinar que, al no formarse grupos coherentes discretos (Fig. 1), los ejemplares incluidos representan parte de la variabilidad de una única especie que, por el principio de prioridad nomenclatural, debe ser llamada Cortaderia hieronymi, como se detalla seguidamente.

\section{Tratamiento taxonómico}

Cortaderia hieronymi (Kuntze) N. P. Barker \& H. P. Linder, Ann. Missouri Bot. Gard. 97: 342. 2010. Triraphis hieronymi Kuntze, Revis. Gen. Pl. 3(3): 373. 1898. Danthonia hieronymi (Kuntze) Hack., Anales Mus. Nac. Buenos Aires 13: 484. 1906. Lamprothyrsus hieronymi (Kuntze) Pilg., Bot. Jarb. Syst. 37, Beibl. 85: 58. 1906. Tipo: Argentina, Córdoba, 06-XI-1881, G. H. E. W. Hieronymus s.n. (Lectotypus B, designado por Conert, Syst. Anat. Arundineae: 128. 1961). Argentina, Córdoba, prope urbem, 06-VI-1881, G. H. E. W. Hieronymus s.n. (Isosintypus CORD).

Lamprothyrsus hieronymi (Kuntze) Pilg. var. nervosus Pilg., Bot. Jahrb. Syst. 37: 59. 1906. Tipo: Argentina, Córdoba, al norte de la cuesta de Copina; Sierra Achala, 11-XI-1878, G. H. E. W. Hieronymus 43 (Holotypus B cb 100272938 imagen!; Isotypus CORD cb 1698 imagen!).

Lamprothyrsus hieronymi (Kuntze) Pilg. var. jujuyensis (Kunze) Pilg., Bot. Jahrb. Syst. 37: 59. 1906. Triraphis hieronymi Kuntze var. jujuyensis Kuntze, Revis. Gen. Pl. 3(3): 374. 1898. Tipo: Argentina, Jujuy, VIII-1892, O. Kuntze s.n. (Holotypus B, fide Conert, Syst. Anat. Arundineae, 129. 1961).

Lamprothyrsus hieronymi (Kuntze) Pilg. var. pyramidatus Pilg., Bot. Jahrb. Syst. 37: 59. 1906. Tipo: Bolivia: Toldas bei Bermejo, 2000 m, 08XII-1903, K. A. G. Fiebrig 2372 (Holotypus B cb 100249138 imagen!).

Lamprothyrsus hieronymi (Kuntze) Pilg. var. tinctus Pilg., Bot. Jahrb. Syst. 37: 59. 1906. Tipo: Bolivia, Bermejo; steiler schiefriger Hang; (tief in Risse eindringend), 1400 m, 16-XII-1903, K. A. G. Fiebrig 2099 (Holotypus B cb 100249137 imagen!; Isotypus $\mathrm{E}$ cb 57467 imagen!).

Lamprothyrsus peruvianus Hitchcock, Proc. Biol. Soc. Washington 36: 195. 1923, syn. nov. Cortaderia peruviana (Hitch.) N. P. Baker \& H. P. Linder, Ann. Missouri Bot. Gard. 97: 342. 2010. Tipo. Perú, Yanahuanca, 16-VI-1922, J. F. Macbride \& W. Featherstone 1205 (Holotypus F cb 40645 imagen!; Isotypus B cb 100249136 imagen!).

Lamprothyrsus venturii Conert, Syst. Anat. Arundineae: 130. 1961. Tipo: Argentina, Tucumán, Famaillá, camino a Villa Nougues, 21-10-1923, S. Venturi 2534 (Holotypus K cb 308609 imagen!; Isotypus US cb 1106104 imagen!, B cb 100272939 imagen!, LIL cb 074 imagen!; SI cb 1442 imagen!; SI cb 1443 imagen!).

Iconografia: Bernardello, 1979: fig. 2 у 3; Lægaard, 1997: fig. 5.

Hierba cespitosa, en matas robustas de 0,5-1,6 $\mathrm{m}$ alt., follaje abundante, tendido; cañas floríferas escasas a numerosas en cada planta, que sobrepasan el follaje; en poblaciones únicamente pistiladas (apomícticas), excepionalmente dioicas. Hojas con vaina frágil, que se fisura tranversalmente, por lo que la lámina cae con una parte de ella y otra parte permamanece enrollada en la base de la planta; lígula corta, 1-2 mm long., densamente pilosa; lámina convoluta o plana, terminada en punta fina. Panojas de 15-60 cm long., nutantes, blancogrisáceas a doradas, brillantes. Espículas 5-12 floras, 8-20 mm long. (excluyendo las aristas), con 
el antecio superior rudimentario. Glumas de 6-8 mm long., enerves o 1-nerves, enteras o cortamente bífidas en el ápice. Callos pilosos, con pelos de 1-3 mm long. Lemas 5-nerves (excepcionalmente 7-nerves), pilosas en el dorso (monomórficas en espiguillas pistiladas y estaminadas), 3-aristadas, aovadas, con la porción basal (cuerpo) indivisa y ápice 2-dentado, prolongado en dos aristas planas y frágiles, y una arista central, mayor, apenas retorcida y robusta, que nace perpendicularmente entre los dientes; lema basal con cuerpo de 2-4,5 mm long., aristas laterales de 10-25 mm y arista central de 16-35 mm long.. Páleas 2-carinadas, iguales o algo mayores que el cuerpo de sus lemas, enteras o cortamente bífidas en el ápice, con pelos diminutos sobre las carinas. Lodículas 2 , cortamente ciliadas. Flores estaminadas con 3 estambres, anteras anchas de $2 \mathrm{~mm}$ long. $\mathrm{y}$ pistilodio pequeño. Flores pistiladas con 3 estaminodios pequeños, blanquecinos, de longitud variable, generalmente mayores que las lodículas. Cariopsis ovoides 2-3 mm long.

Distribución y hábitat: común, desde el sur de Ecuador hasta el hasta el centro de la Argentina, en laderas montañosas de 500 a 3200 m s.n.m.

Material estudiado: ARGENTINA. Prov. Catamarca: Dpto. Pomán, quebrada de Pomán, desde Pampa Chuela hasta el Alto de la Cruz y desde esta hasta Pomán, 31-XII-1904, Spegazzini s.n. (SI 15403). Prov. Córdoba: Dpto. Punilla, Copina, Pampa de Achala, 28-X-1972, Villamil \& Cáceres 187 (BBB); ídem, 07-XII-1974, Villamil \& Cáceres 765 (BBB). Prov. Jujuy: dpto. Capital, laguna de Yala (1200 m s.n.m.), 26-IX-1938, Eyerdam et al. 22224 (SI 84251). Prov. La Rioja: Dpto. Belgrano, Sierra de los Llanos (sector Los Quinteros), subida de Paca Tala desde el paraje la cañada (1660 m s.n.m.), 08-II-2002, Biurrum et al. 7015 (SI). Dpto. Capital, mina El Contadero (2000 m s.n.m.), 28-XII-1941, Meyer 5954 (SI). Prov. Salta: Dpto. Anta, Parque Nacional El Rey, barranca sobre el río Popayán, 12-XI-1983, Cusato 3086 (BAA). Dpto. Cachi, descent from Piedra del Molino, rocky cliff overhanging road (2700 m s.n.m.), 10-XI-1978, Renvoize et al. 3417 (SI). Dpto. La Caldera, La Caldera-El Carmen, ruta 9, km 14 (2430'46”S 65²0’34”W; 1550 m s.n.m.), 07-X-2001, Mello-Silva et al. 18625 (SI). Dpto.
La Viña, Coronel Moldes, La Hoyada, 14-XI-1942, Burkart 13041 (SI). Dpto. Rosario de Lerma, Campo Quijano, $2 \mathrm{~km} \mathrm{~S}$, en camino al Alisal, quebrada del río Toro, 03-XII-1992, Pensiero \& Marino 4231 (SI). Prov. San Juan: Dpto. Valle Fértil, sierra de Elizondo, gruta de la Virgen (1200 m s.n.m.), 16-XII-1987, Múlgura et al. 780 (SI). Prov. Tucumán: Dpto. Burruyacu, Cerro del Campo (1000 m s.n.m.), 24-XI-1928, Venturi 7572 (SI). Dpto. Chicligasta, estancia Las Pavas (900 m s.n.m.), 08-XII-1925, Venturi 3972 (SI). Dpto. Famaillá, quebrada de Lules (800 m s.n.m.), 21 XI-1920, Venturi 1061 (SI). Dpto. Yerba Buena, cerro San Javier (2650'39”'S 65²2'45'W; 1400 m s.n.m.), 10-III-2011, Testoni \& Villamil 386 (BBB).

BOLIVIA. Dpto. La Paz, entre Sorata y Consata (2700 m s.n.m.), 17-XI-1981, Zuloaga \& Vázquez Ávila 1740 (SI). Dpto. Tarija, camino a Yacuiba, $72 \mathrm{~km}$ SE de Entre Ríos, 12-XII-1992, Pensiero \& Marino 4476 (SI). Dpto. Santa Cruz, camino de Santa Cruz a Cochabamba, entre El Torno y Bermejo, 28-IX-1982, Cabrera \& Gutiérrez 33785 (SI).

ECUADOR. Prov. Loja, San Pedro de la Bendita, ruta E35, km 975 (0358'07'S 79²7' 02'W; 2200 m s.n.m.), 12-IV-2012, Testoni \& Villamil 478 (BBB); camino viejo Loja-Cuenca, sobre barrancas del río Zamora (0354’02"S 79¹3'36"; 2000 m s.n.m.), 14-IV-2012, Testoni \& Villamil 507 (BBB); Catamayo-Loja, ca. $1 \mathrm{~km}$ above juntion to the old road, 15-XI-1998, Lægaard 19238 (LOJA);

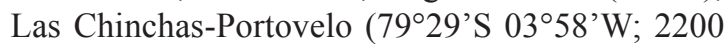
m s.n.m.), 12-IX-1998, Lægaard \& Elizalde 19124 (QCA); Along road Yangana-Cerro Toledo (79¹0'S $04^{\circ} 23^{\prime} \mathrm{W}$; 2150 m s.n.m.) 27-III-1985, Lægaard 53702 (LOJA).

PERÚ. Dpto. Cajamarca, antes de San Cristóbal, camino a Catache (900 m s.n.m.), 07-VI-1986, Llatas Quiroz 1886 (SI); Peña Rota, camino a Cochabamba (2800 m s.n.m.), sine data, Ochora 1566 (SI).

Obs. 1: nombres vernáculos "sevinguilla", "sivinga".

Obs. 2: debido a las pocas veces que se han hallado pies estaminados, se desconoce si el ovario forma cariopsis viables como en otras especies del género; de ser así debería considerarse ginodioca. 


\section{Testoni - Cortaderia peruviana, nuevo sinónimo de C. hieronymi}

\section{Agradecimientos}

A los curadores de los herbarios, al CONICET, a Antonio Garayalde, Carlos B. Villamil y a dos revisores anónimos por sus valiosas sugerencias.

\section{Bibliografía}

BARKER, N. P., H. P. LINDER, C. M. MORTON \& L. MARK. 2003. The paraphyly of Cortaderia (Danthonioideae; Poaceae): evidence from morphology and chloroplast and nuclear DNA sequence data. Ann. Missouri Bot. Gard. 90: 1-24.

BERNARDELLO, L. M. 1979. Sobre el género Lamprothyrsus (Poaceae) en Argentina. Kurtziana 12-13: 119-132.

CONERT, H. J. 1961. Die systematik und anatomie der Arundineae. J. Cramer, Weinheim.

CONNOR, H. E. \& M. I. DAWSON. 1993. Evolution of reproduction in Lamprothyrsus (Arundineae: Gramineae). Ann. Missouri Bot. Gard. 80: 512-517.

DI RIENZO, J. A., F. CASANOVES, M. G. BALZARINI, L. GONZALEZ, M. TABLADA \& C. W. ROBLEDO. 2008. InfoStat. Versión 2008. Grupo InfoStat. FCA, Universidad Nacional de Córdoba, Argentina. Disponible en: http://www.infostat.com. ar

HITCHCOCK, A. S. 1923. New species of grasses from South America. Proc. Biol. Soc. Wash. 36: 195-198.

HITCHCOCK, A. S. 1927. The grasses of Ecuador, Peru, and Bolivia. Contr. U.S. Natl. Herb. 24: 291-556.

INDEX KEWENSIS. 1997. On compact disc version 2.0 for the IBM PC. Oxford University Press, New York.

IPNI, THE INTERNATIONAL PLANT NAMES INDEX. 2010. Published on the Internet http://www. ipni.org [acceso 2015].

LÆGAARD, S. 1997. Gramineae (part 1). En: G. HARLING \& L. ANDERSSON (eds.), Flora of Ecuador 57: 1-53. University of Götenborg, Riksmuseum, Pontificia Universidad Católica del Ecuador, Göteborg, Stockholm, Quito.

LINDER, H. P., M. BAEZA, N. P. BARKER, C. GALLEY, A. M. HUMPHREYS, K. M. LLOYD, D. A. ORLOVICH, M. D. PIRIE, B. K. SIMON, N. WALSH \& G. A. VERBOOM. 2010. A generic classification of the Danthonioideae (Poaceae). Ann. Missouri Bot. Gard. 97: 306-364.
MCNEILL, J., F. R. BARRIE, W. R. BUCK, V. DEMOULIN, W. GREUTER, D. L. HAWKSWORTH, P. S. HERENDEEN, S. KANPP, K. MARHHOLD, J. PRADO, W. F. PRUD'HOMME VAN REINE, G. F. SMITH, J. H. WIERSEMA \& N. J. TURLAND. 2012. International Code of Nomenclature for algae, fungi and plants (Melbourne Code): adopted by the Eighteenth International Botanical Congress Melbourne, Australia, July 2011. Regnum Vegetabile 154. Koeltz Scientific Books, Königstein.

PILGER, R. 1906. Gramineae andinae II. In: URBAN, I. (ed.), Plantae novae andinae imprimis Weberbauerianae I. Bot. Jahrb. Syst. 37: 373-381.

R CORE TEAM. 2013. R: A language and environment for statistical computing. R Foundation for Statistical Computing, Vienna. Disponible en: http://www.Rproject.org/.

SPSS INC. 2006. SPSS for Windows, Version 16.0. SPSS Inc., Chicago.

TESTONI, D. 2015. Estudios en el género Cortaderia (Poaceae). II. Cortaderia atacamensis, nuevo sinónimo de C. speciosa. Bol. Soc. Argent. Bot. 50: 115-119.

TESTONI, D. \& C. B. VILLAMIL. 2014. Estudios en el género Cortaderia (Poaceae). I. Sistemática y nomenclatura de la sect. Cortaderia. Darwiniana, $n$. s. 2: $260-276$.

THIERS, B. Index Herbariorum: a global directory of public herbaria and associated staff. New York Botanical Garden's Virtual Herbarium. Disponible en: http://sweetgum.nybg. org/ih [acceso 2015].

TOVAR, O. 1993. Las Gramíneas (Poaceae) del Perú. Ruizia 13: 1-482.

Recibido el 28 de setiembre de 2015, aceptado el 3 de febrero de 2016. 
